\title{
Efficient Estimation of Extreme Non-linear Roll Motions using the First-order Reliability Method (FORM)
}

Jensen, Jørgen Juncher

Published in:

Marine Science and Technology

Link to article, DOI:

10.1007/s00773-007-0243-z

Publication date:

2007

Document Version

Publisher's PDF, also known as Version of record

Link back to DTU Orbit

Citation (APA):

Jensen, J. J. (2007). Efficient Estimation of Extreme Non-linear Roll Motions using the First-order Reliability Method (FORM). Marine Science and Technology, 12(4), 191-202. https://doi.org/10.1007/s00773-007-0243-z

\section{General rights}

Copyright and moral rights for the publications made accessible in the public portal are retained by the authors and/or other copyright owners and it is a condition of accessing publications that users recognise and abide by the legal requirements associated with these rights.

- Users may download and print one copy of any publication from the public portal for the purpose of private study or research.

- You may not further distribute the material or use it for any profit-making activity or commercial gain

- You may freely distribute the URL identifying the publication in the public portal 


\title{
Efficient estimation of extreme non-linear roll motions using the first-order reliability method (FORM)
}

\author{
Jørgen Juncher Jensen
}

Received: August 29, 2006 / Accepted: February 16, 2007

(C) JASNAOE 2007

\begin{abstract}
In on-board decision support systems, efficient procedures are needed for real-time estimation of the maximum ship responses to be expected within the next few hours, given online information on the sea state and user-defined ranges of possible headings and speeds. For linear responses, standard frequency domain methods can be applied. For non-linear responses, as exhibited by the roll motion, standard methods such as direct time domain simulations are not feasible due to the required computational time. However, the statistical distribution of non-linear ship responses can be estimated very accurately using the first-order reliability method (FORM), which is well known from structural reliability problems. To illustrate the proposed procedure, the roll motion was modelled by a simplified non-linear procedure taking into account non-linear hydrodynamic damping, time-varying restoring and wave excitation moments, and the heave acceleration. Resonance excitation, parametric roll, and forced roll were all included in the model, albeit with some simplifications. The result is the mean out-crossing rate of the roll angle together with the most probable wave scenarios (critical wave episodes), leading to user-specified specific maximum roll angles. The procedure is computationally very effective and can thus be applied to real-time determination of ship-specific combinations of heading and speed to be avoided in the actual sea state.
\end{abstract}

J.J. Jensen $(\bowtie)$

Department of Mechanical Engineering, Technical University of Denmark, Nils Koppel Allé, Building 403/024, Kgs. Lyngby,

DK 2800, Denmark

e-mail: jjj@mek.dtu.dk
Key words Roll motion - Parametric roll · FORM · Reliability $\cdot$ Mean out-crossing rate $\cdot$ On-board decision support systems

\section{Introduction}

The roll motion of ships can lead to various types of failures, ranging from seasickness, cargo shift, and loss of containers to capsize of the vessel. Hence, it is important to minimise the roll motion during a voyage. Currently, on-board decision support systems, e.g., those described by Rathje ${ }^{1}$ and Nielsen et al., ${ }^{2}$ are being installed in vessels with the aim of providing the officer on watch with guidance on the best possible route, taking into account the weather forecast, the time constraints for the voyage, and the limiting criteria for motions, accelerations, and loads.

A major problem is real-time estimation of the sea state. Here, two approaches are tested in full-scale scenarios. The first is based on the use of a wave radar, e.g., the WAVEX system (Borge et al. ${ }^{3}$ ), and the second uses ship responses (e.g., motions, accelerations, and strains) measured in real time by sensors installed on board together with linear transfer functions to estimate the sea state, see the work of Nielsen, ${ }^{4}$ in which a comparison between the two approaches is also found.

After estimation of the sea state, a real-time estimation of the maximum ship responses within the next few hours as a function of ship speed and course is needed to guide the officer on the action to take if excessive responses are foreseen with the present course and speed. For linear responses, the standard frequency domain approach using transfer functions can easily be applied. For non-linear responses, time domain simulations are 
often performed to obtain short-term statistics for the non-linear roll response of ships, e.g., see the work of Krüger et al. ${ }^{5}$ and Daalen et $a{ }^{6}{ }^{6}$ However, less timeconsuming stochastic procedures have also been suggested. Most of these procedures are based on simplifying, but often reasonable, assumptions such as equivalent linear damping (Bulian and Francescutto ${ }^{7}$ ), second- or third-order perturbation procedures (Neves and Rodriguez $^{8}$ ), Melnikov functions (Hsieh at al. ${ }^{9}$ and Spyrou $^{10}$ ), and moment closure techniques (Ness et al. ${ }^{11}$ ).

In the present study, the first-order reliability method (FORM) was applied instead and was shown to be an accurate and efficient procedure for estimation of the extreme value statistics of even very non-linear responses such as parametric rolling. The advantage of the present procedure is that assumptions in the physical model are not needed, as full three-dimensional non-linear hydrodynamic codes can be applied within the FORM analysis. Furthermore, in contrast to Monte Carlo simulations, the calculation time in the FORM analysis does not depend very much on the exceedance level considered and thus even very rare events can be predicted within a short calculation time.

A problem related to on-board decision support systems is the estimation of limit state parameters for the ship. This involves a discussion of the various hazards: seasickness, destruction of sensible equipment, cargo shift, loss of containers, and, ultimately, the capsizing or sinking of the vessel. Clearly, both a model for the failure mode and a cost model for the consequences are needed to define a proper limiting value to be presented to the officer on watch. A general procedure based on a socalled utility function has been suggested by Søborg and Friis-Hansen. ${ }^{12}$ In the present work, a simplified model for the roll motion was used to calculate only the exceedance level of specific roll angles. Thus, no attempt to formulate a realistic limiting roll angle or to define a suitable utility function was made. It should, however, be mentioned that such limit state parameters and criteria were included in the SAFEDOR project, see the Acknowledgment, and can easily be implemented in the present FORM approach.

\section{First-order reliability method applied to wave loads}

\subsection{Design point and reliability index}

In FORM analysis, the excitation or input process is a stationary stochastic process. Considering wave loads on marine structures in general, the input process is the wave elevation and the associated wave kinematics. For moderate sea states, the wave elevation can be consid- ered as Gaussian distributed, whereas for severer wave conditions, corrections for non-linearities must be incorporated. Such corrections are discussed and accounted for by using a second-order wave theory in a FORM analysis of a jack-up platform (Jensen and Capul ${ }^{13}$ ). In the present work with the roll motion of a ship, linear, long-crested waves are assumed and hence the normal distributed wave elevation $H(X, t)$ as a function of space $X$ and time $t$ can be written:

$$
H(X, t)=\sum_{i=1}^{n}\left(u_{i} c_{i}(X, t)+\bar{u}_{i} \bar{c}_{i}(X, t)\right)
$$

where the variables $u_{i}, \bar{u}_{i}$ are uncorrelated, standard normally distributed variables to be determined by the stochastic procedure and with the deterministic coefficients given by:

$$
\begin{aligned}
& c_{i}(x, t)=\sigma_{i} \cos \left(\omega_{i} t-k_{i} X\right) \\
& \bar{c}_{i}(x, t)=-\sigma_{i} \sin \left(\omega_{i} t-k_{i} X\right) \\
& \sigma_{i}^{2}=S\left(\omega_{i}\right) d \omega_{i}
\end{aligned}
$$

where $\omega_{i}, k_{i}=\omega_{i}^{2} / g$ are the $n$ discrete frequencies and wave numbers applied. Furthermore, $S(\omega)$ is the wave spectrum and $d \omega_{i}$ the increment between the discrete frequencies. It is easily seen that the expected value $E\left[H^{2}\right]=$ $\int S(\omega) d \omega$, thus the wave energy in a stationary sea is preserved. Short-crested waves could be incorporated, if needed, but require more unknown variables $u_{i}, \bar{u}_{i}$.

From the wave elevation (Eqs. 1 and 2) and the associated wave kinematics, any non-linear wave-induced response $\phi(t)$ of a marine structure can in principle be determined by a time domain analysis using a proper hydrodynamic model:

$\phi=\phi\left(t \mid u_{1}, \bar{u}_{1}, u_{2}, \bar{u}_{2}, \ldots, u_{n}, \bar{u}_{n}\right.$, initial conditions $)$

Each of these realisations represents the response for a possible wave scenario. The influence of the initial conditions should be eliminated as stationary stochastic responses are considered. The realisation which exceeds a given threshold $\phi_{0}$ at time $t=t_{0}$ with the highest probability is sought. This problem can be formulated as a limit state problem, which is well known within timeinvariant reliability theory (Der Kiureghian ${ }^{14}$ ):

$$
\begin{aligned}
& G\left(u_{1}, \bar{u}_{1}, u_{2}, \bar{u}_{2}, \ldots, u_{n}, \bar{u}_{n}\right) \\
& \quad \equiv \phi_{0}-\phi\left(t_{0} \mid u_{1}, \bar{u}_{1}, u_{2}, \bar{u}_{2}, \ldots, u_{n}, \bar{u}_{n}\right)=0
\end{aligned}
$$

The integration in Eq. 4 must cover a sufficient time period $\left\{0, t_{0}\right\}$ to avoid any influence on $\phi\left(t_{0}\right)$ of the initial conditions at $t=0$, i.e., the integration period must be longer than the memory in the system. Proper values of 
$t_{0}$ would usually be $1-3 \mathrm{~min}$, depending on the damping in the system. Hence, to avoid repetition in the wave system and for accurate representation of typical wave spectra, $n=15-50$ is suitable.

An approximate solution can be obtained using FORM. The limit state surface $G$ is given in terms of the uncorrelated standard normally distributed variables $\left\{u_{i}, \bar{u}_{i}\right\}$, and hence determination of the design point $\left\{u_{i}^{*}\right.$, $\left.\bar{u}_{i}^{*}\right\}$, defined as the point on the failure surface $G=0$ with the shortest distance to the origin, is rather straightforward. A linearization around this point replaces Eq. 4 with a hyperplane in $2 \mathrm{n}$ space. The distance $\beta_{F O R M}$

$$
\beta_{F O R M}=\min \sqrt{\sum_{i=1}^{n}\left(u_{i}^{2}+\bar{u}_{i}^{2}\right)}
$$

from the hyperplane to the origin is denoted the FORM reliability index. The calculation of the design point $\left\{u_{i}^{*}\right.$, $\left.\bar{u}_{i}^{*}\right\}$ and the associated value of $\beta_{F O R M}$ can be performed by standard reliability codes (e.g., that of Det Norske Veritas $^{15}$ ). Alternatively, standard optimisation codes using Eq. 5 as the objective function and Eq. 4 as the constraint can be applied.

The deterministic wave profile

$$
H^{*}(X, t)=\sum_{i=1}^{n}\left(u_{i}^{*} c_{i}(X, t)+\bar{u}_{i}^{*} \bar{c}_{i}(X, t)\right)
$$

can be considered as a design wave or a critical wave episode. It is the wave scenario with the highest probability of occurrence that leads to the exceedance of the specified response level $\phi_{0}$. For linear systems, the result reduces to the standard Slepian model, see e.g., Lindgren, ${ }^{16}$ Tromans et al., ${ }^{17}$ Adegeest et al., ${ }^{18}$ and Dietz et al. ${ }^{19}$ The critical wave episode is a useful result as it can be used as input in more elaborate time domain simulations to correct for assumptions made in the hydrodynamic code, Eq. 3, applied in the FORM calculations. Such a model-correction-factor approach provides an effective tool for accounting for even very complicated non-linear effects (Ditlevsen and ArnbjergNielsen ${ }^{20}$ ).

It should be noted that other definitions of design waves based on a suitable non-uniform distribution of phase angles have been applied, especially for experimental application in model basins. The selection of the phase angle distribution is, however, not obvious, see e.g., Alford et al. ${ }^{21}$
2.2 Mean out-crossing rates and exceedance probabilities

The time-invariant peak distribution follows from the mean out-crossing rates. Within a FORM approximation, the mean out-crossing rate can be written as follows (see Jensen and Capul ${ }^{13}$ ):

$v\left(\phi_{0}\right)=\frac{1}{2 \pi \beta_{F O R M}} e^{-\frac{1}{2} \beta_{F O R M}^{2}} \sqrt{\sum_{i=1}^{n}\left(u_{i}^{* 2}+\bar{u}_{i}^{* 2}\right) \omega_{i}^{2}}$

This is based on a general formula given by Koo et al. ${ }^{22}$ Thus, the mean out-crossing rate is expressed analytically in terms of the design point and the reliability index. For linear processes, it reduces to the standard Rayleigh distribution. Often, the gradient vector $\left\{\alpha_{i}^{*}\right.$, $\left.\bar{\alpha}_{i}^{*}\right\}=\left\{u_{i}^{*}, \bar{u}_{i}^{*}\right\} / \beta_{F O R M}$ to the design point does not vary much with exceedance level $\phi_{0}$. Hence, Eq. 7 reduces to:

$v\left(\phi_{0}\right)=v_{0} e^{-\frac{1}{2} \beta_{F O R M}^{2}}$

where $v_{0}$ can be viewed as an effective mean zero outcrossing rate. Finally, on the assumption of statistically independent peaks and, hence, a Poisson distributed process, the probability of exceedance of the level $\phi_{0}$ in a given time $T$ can be calculated from the mean outcrossing rate $v\left(\phi_{0}\right)$ :

$P\left[\max _{T} \phi>\phi_{0}\right]=1-e^{-v\left(\phi_{0}\right) T}$

The present procedure can be considered as an alternative to the random constrained simulation, e.g., see Dietz et al. ${ }^{19}$ The present method has, however, the advantage that the number of time domain simulations is much smaller due to the very efficient optimisation procedures within FORM; in addition, it does not require the curvefitting of lines of constant probabilities needed in the other procedure. Furthermore, the FORM procedure does not rely on a mean wave conditional on a linear response and can hence be applied also to bifurcation types of problems such as parametric roll. In such cases, the optimisation procedure used in the FORM analysis must be chosen appropriately, i.e., it must be of the nongradient type. In the present case, a circle step approach is used, see Det Norske Veritas. ${ }^{15}$ In this approach, a circle is drawn in u-space and the point with the lowest value of $G$ is determined. Then the intersection between the line through origin and this point and $G=0$ is found. In the next iteration, a new circle in drawn through this intersection point and so on. Furthermore, to facilitate the convergence of the optimisation procedure, the limit 
state surface, Eq. 4, is replaced by a logarithm transformation:

$$
\begin{aligned}
& \tilde{G}\left(u_{1}, \bar{u}_{1}, u_{2}, \bar{u}_{2}, \ldots, u_{n}, \bar{u}_{n}\right) \\
& \quad \equiv \operatorname{logt}\left(\phi_{0}\right)-\operatorname{logt}\left(\phi\left(t_{0} \mid u_{1}, \bar{u}_{1}, u_{2}, \bar{u}_{2}, \ldots, u_{n}, \bar{u}_{n}\right)\right)=0 \\
& \operatorname{logt}(y) \equiv\left\{\begin{array}{lc}
-1-\log (-y) ; y<-1 \\
y ; & -1 \leq y \leq 1 \\
1+\log (y) ; & 1<y
\end{array}\right.
\end{aligned}
$$

FORM is significantly faster than direct Monte Carlo simulations, and most often is very accurate. In a study (Jensen and Pedersen ${ }^{23}$ ) dealing exclusively with the parametric rolling of ships in head seas, the FORM approach was found to be two orders of magnitude faster than direct simulation for realistic exceedance levels and with results deviating by less than 0.1 in the reliability index. Similar observations were noted in the present study.

It should be mentioned that the present procedure bears some resemblance to the approach adopted by Søborg and Friis-Hansen. ${ }^{12}$ The difference is basically in the excitation process, where the present continuous wave excitation in Søborg and Friis-Hansen ${ }^{12}$ is replaced by a discrete excitation in time. The advantage of the present procedure is that it can directly represent the shape of actual wave spectra and that it provides an analytical formula, Eq. 7, for the mean out-crossing rate.

\section{Roll motion of a ship}

A very comprehensive discussion of intact stability can be found in a recent ITTC report on ship stability in waves, ITTC. ${ }^{24}$ The report discusses various modes of failure, e.g., capsize, and the prediction procedures available. The report is partly based on the result of a questionnaire distributed to a large number of organisations and thus reflects very well the current status. To cover all modes of failure (static loss of stability, parametric excitation, dynamic rolling, resonance excitation, and broaching) a general, non-linear, six-degrees-offreedom time domain procedure, including viscous effects and manoeuvring models, is probably needed. Some codes, e.g., LAMP (France et al. ${ }^{25}$ and Shin et $\mathrm{al}^{26}$ ), seem to be able to achieve this with reasonable accuracy, but are very time-consuming to run, restricting the application to regular waves or very short stochastic realisations.

Other procedures have more limited capabilities as some of the capsize modes are excluded. An example is the ROLLS procedure (Kroeger ${ }^{27}$ ), in which the follow- ing non-linear differential equation is used to estimate the roll angle $\phi(t)$, omitting the terms due to wind and fluids in tanks:

$$
\begin{aligned}
& \left(I_{x x}-I_{x z}(\psi \sin \phi+\theta \cos \phi)\right) \ddot{\phi}=M_{\phi}+M_{s y}-M_{d} \\
& \quad-\Delta(g-\ddot{w}) G Z(\phi)-I_{x z}\left[\left(\ddot{\theta}+\theta \dot{\phi}^{2}\right) \sin \phi\right. \\
& \left.\quad-\left(\ddot{\psi}+\psi \dot{\phi}^{2}\right) \cos \phi\right]
\end{aligned}
$$

Here, $M_{\phi}, M_{s y}$, and $M_{d}$ are the roll moments due to waves, sway and yaw, and hydrodynamic damping, respectively. Furthermore, $I_{x x}$ and $I_{x z}$ are the mass moment of inertia about the longitudinal axis and the cross-term mass moment of inertia. The coordinate system is situated with the origin at the centre of gravity. The displacement of the ship is denoted by $\Delta$, and $g$ is the acceleration of gravity. The instantaneous value of the righting arm, $G Z$, in irregular waves is calculated approximately using the so-called Grim's effective wave. The heave $w$, pitch $\theta$, and yaw $\psi$ motions are determined by standard strip theory formulations, whereas the surge motion is calculated from the incident wave pressure distribution. The advantage of this formulation compared to full non-linear calculations is the much faster computational speed, while still retaining a coupling between all six degrees of freedom, see Krüger et al. ${ }^{5}$ The model, however, cannot deal with broaching.

In the present procedure, both the heave motion $w$ and the wave-induced roll moment $M_{\phi}$ are taken to be linear functions of the wave elevation. The cross-term mass moment of inertia is assumed to be small, and pitch is thus only included through the static balancing of the vessel in waves in the calculation of the $G Z$ curve. Furthermore, the sway, yaw, and surge motions are ignored because the vertical motions have the largest influence on the instantaneous $G Z$ curve. The damping term $M_{d}$ is modelled by a standard combination of a linear, a quadratic, and a cubic variation in the roll velocity. With these simplifications, Eq. 11 becomes:

$$
\ddot{\phi}=-2 \beta_{1} \omega_{\phi} \dot{\phi}-\beta_{2} \dot{\phi}|\dot{\phi}|-\frac{\beta_{3} \dot{\phi}^{3}}{\omega_{\phi}}-\frac{(g-\ddot{w}) G Z(\phi)}{r_{x}^{2}}+\frac{M_{\phi}}{I_{x x}}
$$

where $r_{x}$ is the roll radius of gyration. The roll frequency $\omega_{\phi}$ is given by the metacentric height $G M_{s w}$ in still water:

$\omega_{\phi}=\frac{\sqrt{g G M_{s w}}}{r_{x}}$

It is clear that this model is very simplistic, but it is well suited to illustrate the proposed stochastic procedure as it can model parametric rolling, resonance excitation, and forced rolling. Hence, it is possible to identify which 
mode is the most probable for a given combination of sea state, speed, and heading. As broaching and dynamic rolling (where a strong coupling to surge exists) cannot be modelled by Eq. 12, following and stern quartering seas will be excluded from the calculations, and heading angles $\psi$ in the range $60^{\circ}-180^{\circ}$ (head sea) only will be considered.

The instantaneous $G Z$ curve in irregular waves was estimated from numerical results for a regular wave with a wavelength equal to the length $L$ of the vessel and a wave height equal to $0.05 \mathrm{~L}$. These numerical results were fitted with analytical approximations of the form:

$$
\begin{aligned}
G Z\left(\phi, x_{c}\right)= & \left(C_{0} \sin \phi+C_{1} \phi+C_{3} \phi^{3}+C_{5} \phi^{5}\right) \cos ^{4}\left(\frac{\pi x_{c}}{L_{e}}\right) \\
& +\left(D_{0} \sin \phi+D_{1} \phi+D_{3} \phi^{3}+D_{5} \phi^{5}\right) \sin \left(\frac{\pi x_{c}}{L_{e}}\right)
\end{aligned}
$$

where the wave crest position $x_{c}$ is measured relative to the aft end of the vessel. Similarly, the $G Z$ curve in still water was fitted by:

$$
G Z_{s w}(\phi)=\left(G M_{s w}-A_{1}\right) \sin \phi+A_{1} \phi+A_{3} \phi^{3}+A_{5} \phi^{5}
$$

The coefficients $\left(A_{1}, A_{3}, A_{5}, C_{0}, C_{1}, C_{3}, C_{5}, D_{0}, D_{1}, D_{3}, D_{5}\right.$, $\left.L_{e}\right)$ in Eqs. 14 and 15 were found by the least-squares method. Other polynomial or Fourier series representations have been suggested, e.g., those of Spyrou ${ }^{10}$ and Bulian, ${ }^{29}$ and generally a very good fit can be achieved for the range of roll angles of interest.

In a stochastic seaway, the following approximation of the instantaneous value of the righting arm $G Z(t)$ is then applied:

$$
G Z(\phi, t)=G Z_{s w}(\phi)+\frac{h(t)}{0.05 L}\left(G Z\left(\phi, x_{c}(t)\right)-G Z_{s w}(\phi)\right)
$$

This linear relation between $G Z$ and $h$ is clearly an assumption that needs validation. It is used here for the sake of simplicity, but also because the model given in Eq. 12 by itself gives only an approximate description of reality.

The instantaneous wave height $h(t)$ along the length of the vessel and the position of the crest $x_{c}$ are deter- mined by an equivalent wave procedure somewhat similar to the one used by Kroeger ${ }^{27}$ :

$$
\begin{aligned}
& a(t)=\frac{2}{L_{e}} \int_{0}^{L_{e}} H(X(x, t), t) \cos \left(\frac{2 \pi x}{L_{e}}\right) d x \\
& b(t)=\frac{2}{L_{e}} \int_{0}^{L_{e}} H(X(x, t), t) \sin \left(\frac{2 \pi x}{L_{e}}\right) d x \\
& X(x, t)=(x+V t) \cos \psi \\
& h(t)=2 \sqrt{a^{2}(t)+b^{2}(t)} \\
& x_{c}(t)= \begin{cases}\frac{L_{e}}{2 \pi} \arccos \left(\frac{2 a(t)}{h(t)}\right) & \text { if } b(t)>0 \\
L_{e}-\frac{L_{e}}{2 \pi} \arccos \left(\frac{2 a(t)}{h(t)}\right) & \text { if } b(t)<0\end{cases}
\end{aligned}
$$

Note than in beam seas, $h(t)=0$ such that $G Z(\phi, t)=$ $G Z_{s w}(\phi)$. Stationary sea conditions were assumed and were specified by a JONSWAP wave spectrum with significant wave height $H_{s}$ and zero-crossing period $T_{z}$. The frequency range was taken to be $\pi \leq \omega T_{z} \leq 3 \pi$, covering the main part of the JONSWAP spectrum.

Solutions were obtained by embedding the time domain simulation routine, Eq. 12, in a standard FORM code. In the present case, the software PROBAN (Det Norske Veritas ${ }^{15}$ ) was used. In the following, results for a container ship are presented and discussed.

\section{Numerical example}

A container ship with main particulars given in Table 1 is considered. The damping coefficients, $\beta_{1}-\beta_{3}$, are taken, quite arbitrarily, from a study considering a different vessel $\left(\right.$ Bulian $\left.^{29}\right)$, but correspond to about 0.05 in equivalent linear damping. The speed is chosen such that the mean encounter frequency is close to twice the roll natural frequency. The coefficients in Eqs. 14 and 15 are

\begin{tabular}{|c|c|c|c|c|c|c|c|c|c|}
\hline $\begin{array}{l}\text { Length } \\
L \\
\end{array}$ & $\begin{array}{l}\text { Breadth } \\
B \\
\end{array}$ & $\begin{array}{l}\text { Draught } \\
D \\
\end{array}$ & $\begin{array}{l}\text { Block } \\
\text { coeff. } C_{b}\end{array}$ & $\beta_{1}$ & $\beta_{2}$ & $\beta_{3}$ & $G M_{s w}$ & $\begin{array}{l}\text { Radius of } \\
\text { gyration } r_{x}\end{array}$ & $\begin{array}{l}\text { Speed } \\
V \\
\end{array}$ \\
\hline $284 \mathrm{~m}$ & $32.2 \mathrm{~m}$ & $10.5 \mathrm{~m}$ & 0.61 & 0.012 & 0.40 & 0.42 & $0.89 \mathrm{~m}$ & $0.4 B$ & $6 \mathrm{~m} / \mathrm{s}$ \\
\hline
\end{tabular}
given in Table 2.

The units of the coefficients are metres with the roll angle given in radians. The approximations are accurate for roll angles up to 0.9 radians, see Jensen and Olsen. ${ }^{28}$ It is noted that the optimal value of the parameter $L_{e}$ is slightly shorter than the length of the ship $L$. The $G Z$ curves are shown in Fig. 1 and it is clear that a significant

Table 1. Main particulars of a container ship 
Table 2. Coefficients in the analytical approximations for the righting arm $(\mathrm{GZ})$ curves, Eqs. 14 and 15

Fig. 1. a Righting arm $(G Z)$ curve in still water. b GZ curves in regular waves with the wavelength equal to the ship length $(L)$ and a wave height equal to $0.05 L$. Wave crest positions are at $x_{c}=0$, $0.25 L, 0.5 L$, and $0.75 L$

\begin{tabular}{llllllll}
\hline$A_{1}$ & $A_{3}$ & $A_{5}$ & \multicolumn{1}{c}{$C_{0}, D_{0}$} & $C_{1}, D_{1}$ & \multicolumn{1}{c}{$C_{3}, D_{3}$} & \multicolumn{1}{c}{$C_{5}, D_{5}$} & $L_{e}$ \\
\hline \multirow{2}{*}{10.7964} & \multirow{2}{*}{2.30187} & \multirow{2}{*}{-2.97748} & 2.96515, & 2.06522, & -3.6616, & 0.83496, & 259.2 \\
& & & -0.40240 & 0.83103 & 1.66807 & -1.40701 & \\
\hline
\end{tabular}

(a)

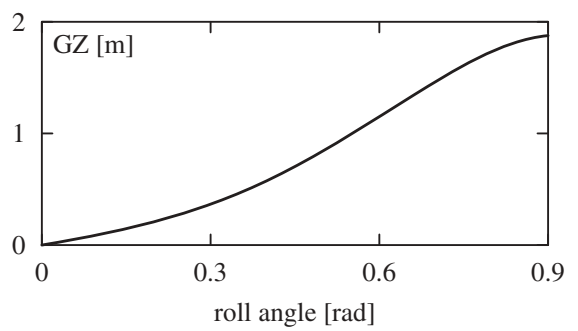

(b)

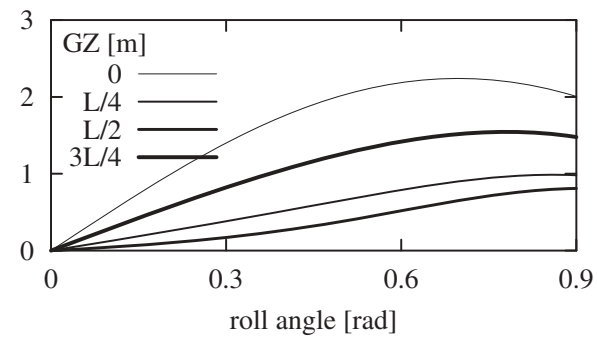

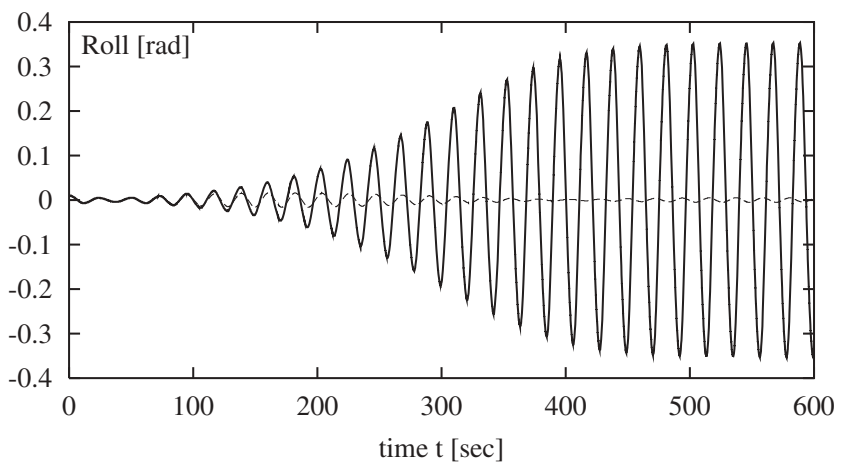

Fig. 2. Parametric roll in a regular wave (solid line) and the roll response for a slightly smaller regular wave (dashed line), from Jensen and Pedersen ${ }^{23}$

reduction in righting lever occurs when the wave crest moves from the aft perpendicular (AP) to $0.25 \mathrm{~L}$ forward of AP. The lowest value of $G Z$ occurs when the wave crest is amidships. This is quite typical for ships with fine hull forms, such as container ships.

By use of the closed-form expressions given in Jensen et al. ${ }^{30}$ for the heave $w$ and the wave-induced roll moment $M_{\phi}$, all pertinent data for calculation of the roll angle as a function of time are defined. In order to show that Eq. 12 can model parametric roll, calculations have been performed with a regular wave with an encounter frequency close to twice the roll frequency, see Jensen and Pedersen. ${ }^{23}$ Two wave heights were used: one $(3.65 \mathrm{~m})$ for which parametric roll is not triggered and one slightly higher wave $(3.7 \mathrm{~m})$ for which parametric roll develops. The roll motions for the two wave heights are shown in Fig. 2. The onset of parametric roll and its saturation level are clearly depicted.

The regular wave height needed to trigger parametric roll is thus about $3.7 \mathrm{~m}$ for the present vessel. If the wave height is increased above this value, parametric roll develops faster and to a higher saturation level. This qualitative behaviour is consistent with both model test results and numerical calculations using more elaborate hydrodynamic codes, see France et al..$^{25}$

In the following discussion, results are shown for a range of combinations of sea states, headings, and forward speeds. The reference sea state has a significant wave height $H_{s}=12 \mathrm{~m}$ and a zero-crossing wave period $T_{z}=11.7 \mathrm{~s}$. The zero-crossing period was chosen such that parametric roll could be expected to develop because the occurrence of encounter frequencies was in the range of twice the roll frequency. Note, however, that neither the encounter frequency nor the roll frequency is constant in irregular waves. The time domain simulations were carried out from $t=0$ to $t=t_{0}=150-300 \mathrm{~s}$. The effect of the initial conditions $[\phi(t=0)=0.01$ radians, $\dot{\phi}(t=0)=0$ ] was negligible after about $50 \mathrm{~s}$, but in order to build up parametric roll, a longer duration was needed. With $n=50$ equidistant frequencies, the wave repetition period relative to the ship was about $400 \mathrm{~s}$, depending on the forward speed. This number of frequencies was used in the first part of the results dealing with parametric roll in head seas, whereas $n=25$ and $t_{0}=180 \mathrm{~s}$ were later shown to be sufficient when the roll response in other headings was considered. In principle, the duration to establish parametric role could be almost infinite, but the use of longer simulation times than 180 s only marginally changed the mean out-crossing rates, and hence the probability of occurrence.

\subsection{Parametric roll in a head sea in a stochastic seaway}

A detailed analysis using the present approach is given in Jensen and Pedersen ${ }^{23}$ for the reference condition [head sea $\left(\psi=180^{\circ}\right), V=6 \mathrm{~m} / \mathrm{s}, H_{s}=12 \mathrm{~m}, T_{z}=11.7 \mathrm{~s}$, 
Fig. 3. a Most probable roll response yielding a limiting roll angle of $\phi_{0}=0.5$ radians at $t_{0}=300 \mathrm{~s}$. b Corresponding critical wave episode amidships (a)

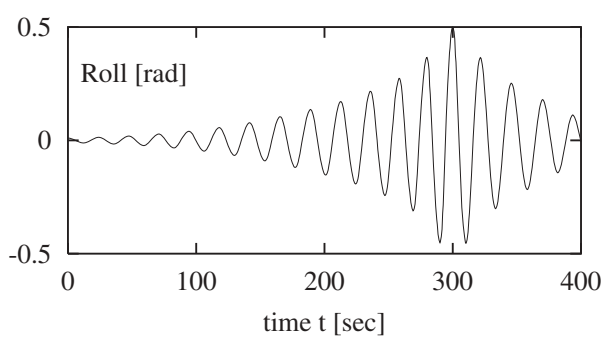

(a)

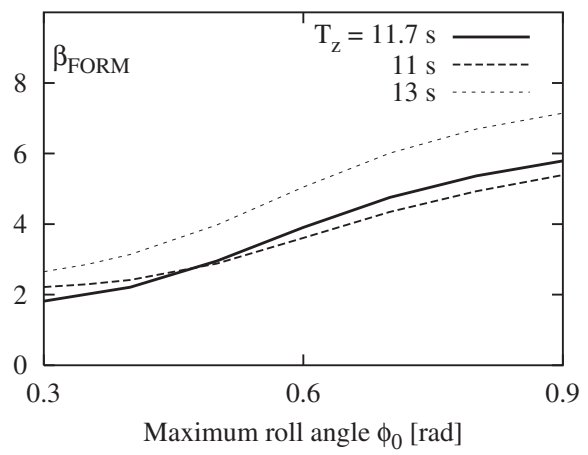

(b)

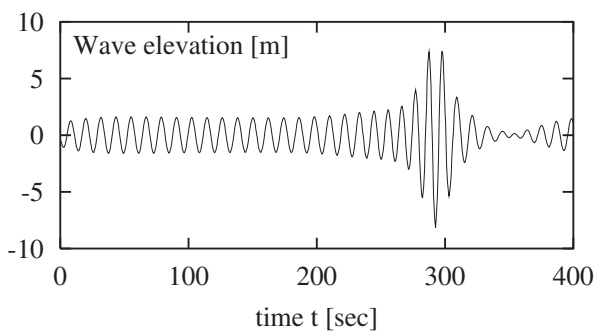

(b)

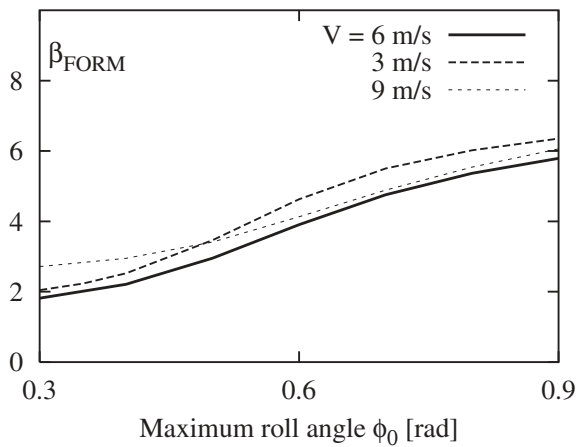

Fig. 4. Reliability index $\beta_{F O R M}$ as a function of limiting roll angle $\phi_{0}$ for different zerocrossing periods $T_{z}$ (a) and ship speeds $V(\mathbf{b})$. Reference case: head sea, $H_{s}=12 \mathrm{~m}$, $T_{z}=11.7 \mathrm{~s}, V=6 \mathrm{~m} / \mathrm{s}, n=50$, and $t_{0}=300 \mathrm{~s}$
Table 3. Reliability index $\beta_{F O R M}$ as a function of integration length $t_{0}$ and number of frequencies $n$

\begin{tabular}{llll}
\hline$t_{0}[\mathrm{~s}]$ & $n$ & $\beta_{F O R M}\left(\phi_{0}=0.3 \mathrm{rad}\right)$ & $\beta_{F O R M}\left(\phi_{0}=0.5 \mathrm{rad}\right)$ \\
\hline 300 & 50 & 1.8146 & 2.9556 \\
180 & 25 & 1.9658 & 3.0447 \\
150 & 25 & 2.2767 & 3.2232 \\
\hline
\end{tabular}

Head sea, $V=6 \mathrm{~m} / \mathrm{s}, H_{s}=12 \mathrm{~m}, T_{z}=11.7 \mathrm{~s}$

the wave spectrum does not change shape with $H_{s}$, the critical wave episode, Eq. 6, becomes independent of $H_{s}$. A change of $H_{s}$ by a factor $\mu$ will then just change the design point $\left\{u_{i}^{*}, \bar{u}_{i}^{*}\right\}$ and hence also $\beta_{\text {FORM }}$ by a factor $1 / \mu$. This is an important observation, because calculations then only have to be done for one value of the significant wave height. It might seem a strange result for non-linear systems, but one has to remember that $\beta_{F O R M}$ is a non-linear function of the maximum roll angle specified. Whereas the critical wave episode is independent of $H_{s}$, the probability that this wave episode will occur depends on $H_{s}$ through $\beta_{F O R M}$. As $\beta_{F O R M}$ decreases with increasing $H_{s}$, the probability of parametric roll decreases with decreasing $H_{s}$. If a change of $H_{s}$ is accompanied by a change in $T_{z}$, the situation is more complex, as discussed below.

The variation with the zero-crossing period $T_{z}$ is shown in Fig. 4a. Increasing $T_{z}$ moves the two-to-one resonance condition away from the dominant encounter sensitivity of the reliability index $\beta_{F O R M}$ to the sea state 
wave and roll resonance periods and hence increases $\beta_{F O R M}$. By lowering $T_{z}$, an increase in $\beta_{F O R M}$ is seen for smaller roll angles, whereas a decrease is noted for higher roll angles. This is due to the $G Z$ dependence of the roll frequency, which implies that the roll resonance period decreases with increasing roll angle.

The variation with ship speed $V$, Fig. $4 \mathrm{~b}$, shows that the probability of parametric roll decreases if the speed is either lowered or increased for the present example. For lower limiting roll angles, it is seen to be better to increase the speed than to reduce it. Finally, it is noted that for a linear system, the reliability index $\beta_{\text {FORM }}$ would be linearly dependent on the limiting roll angle $\phi_{0}$ (with the standard deviation as scale parameter), but this is not the case here.

4.2 Roll response as a function of heading and speed in a stochastic seaway

For other headings than a head sea, the roll moment $M_{\phi}$ due to waves is non-zero and direct resonance and forced roll can be excited as well. Hence, a detailed investigation of the most probable roll mode as a function of heading is made. The two limiting roll angles $\phi_{0}=0.3$ and 0.5 radians are chosen because they could be representative of warning and alarm levels, respectively, in an on-board decision support system.

Even though FORM analysis is fast, a decision support system requires near real-time estimates and thus it is important to minimise the calculation time. From Table 3 a suitable integration length, and number of frequencies seem to be $t_{0}=180 \mathrm{~s}$ and $n=25$ in a head sea. This is further supported by calculations for other heading angles, see Table 4 . It is clear that the memory in the system decreases when other roll modes become active, such that even $t_{0}=150 \mathrm{~s}$ could have been used for headings other than near head sea conditions.

Figure 5 shows the reliability index $\beta_{F O R M}$ as a function of the heading for two speeds: $V=6 \mathrm{~m} / \mathrm{s}$ and $V=$ $2 \mathrm{~m} / \mathrm{s}$. For the present sea condition, head or near head seas yield the lowest reliability index and thus the highest probability of exceeding the given limiting roll angles $\phi_{0}$.
Reducing the speed from 6 to $2 \mathrm{~m} / \mathrm{s}$ is generally beneficial in head sea conditions, but the opposite is the case in the heading range $70^{\circ}-90^{\circ}$ and, for $\phi_{0}=0.5$ radians, also for the heading range $120^{\circ}-150^{\circ}$.

The calculations are done with $5^{\circ}$ steps in heading angle. Some waviness of the curves is observed due to different active roll modes for different heading ranges. This is illustrated in Figs. 6-10, in which the most probable roll responses yielding a limiting angle $\phi_{0}=0.5$ radians at $t_{0}=180 \mathrm{~s}$ together with the associated critical

Table 4. Reliability index $\beta_{F O R M}$ as a function of integration length $t_{0}$

\begin{tabular}{clll}
\hline Heading $\psi$ & $t_{0}(\mathrm{~s})$ & $\beta_{F O R M}\left(\phi_{0}=0.3 \mathrm{rad}\right)$ & $\beta_{F O R M}\left(\phi_{0}=0.5 \mathrm{rad}\right)$ \\
\hline \multirow{2}{*}{$60^{\circ}$} & 150 & 2.7965 & 5.4356 \\
& 180 & 2.7697 & 5.3928 \\
$90^{\circ}$ & 150 & 2.5824 & 4.6737 \\
& 180 & 2.5709 & 4.6713 \\
$120^{\circ}$ & 150 & 2.7716 & 4.5771 \\
& 180 & 2.6926 & 4.4360 \\
$150^{\circ}$ & 150 & 2.3043 & 3.5075 \\
& 180 & 2.1093 & 3.3634
\end{tabular}

$V=6 \mathrm{~m} / \mathrm{s}, H_{s}=12 \mathrm{~m}, T_{z}=11.7 \mathrm{~s}, n=25$

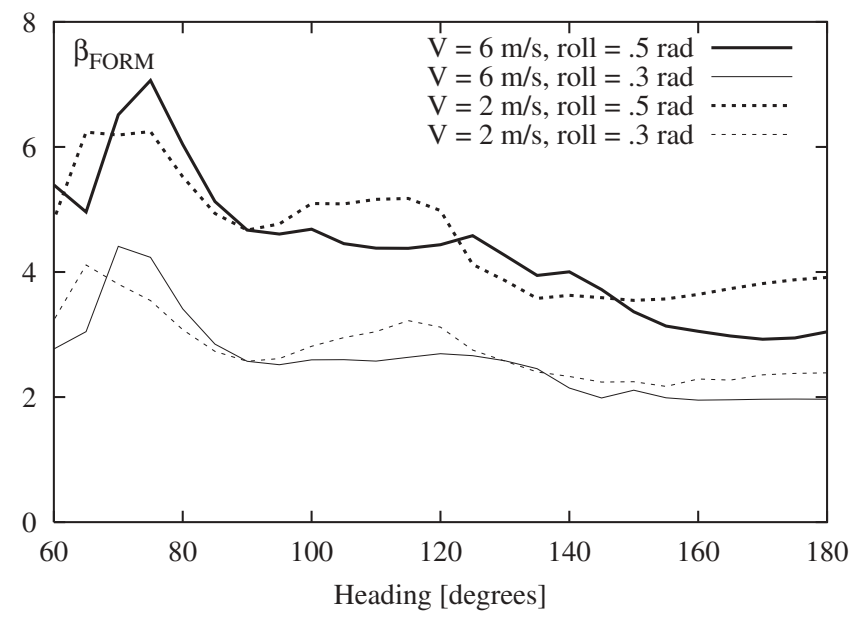

Fig. 5. Reliability index $\left(\beta_{F O R M}\right)$ as a function of heading angle for different limiting roll angles $\phi_{0}$ and two ship speeds $V$. Other parameters: $H_{s}=12 \mathrm{~m}, T_{z}=11.7 \mathrm{~s}, n=25$, and $t_{0}=180 \mathrm{~s}$
Fig. 6. Most probable roll response (a) and critical wave episode amidships (b). Heading $\psi=180^{\circ}$

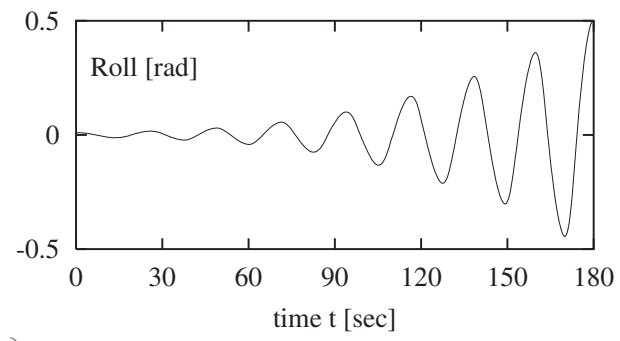

(a)

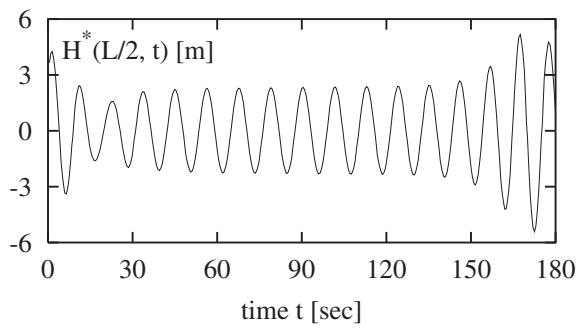


Fig. 7. Most probable roll response (a) and critical wave episode amidships (b). Heading $\psi=150^{\circ}$

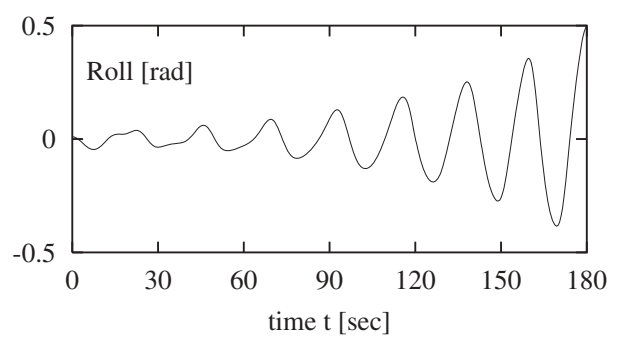

(a)

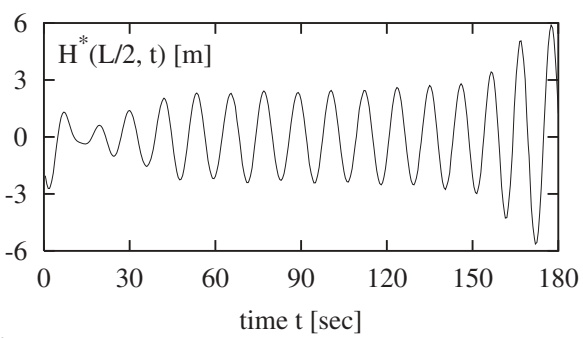

(b)

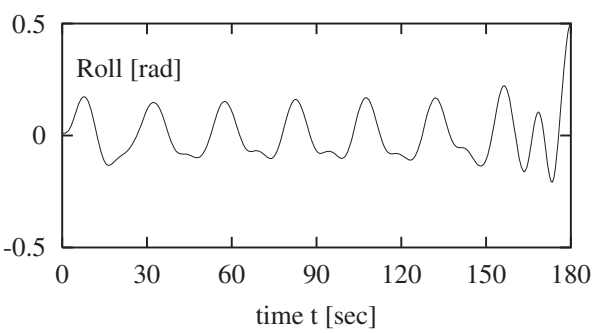

(a)

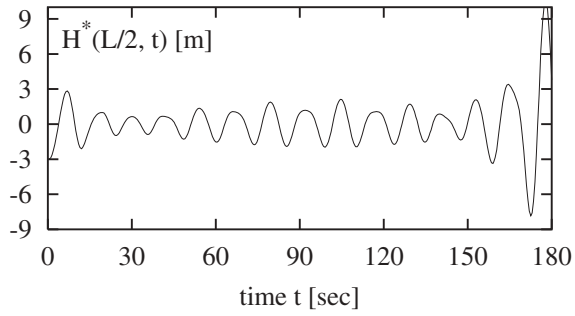

(b)

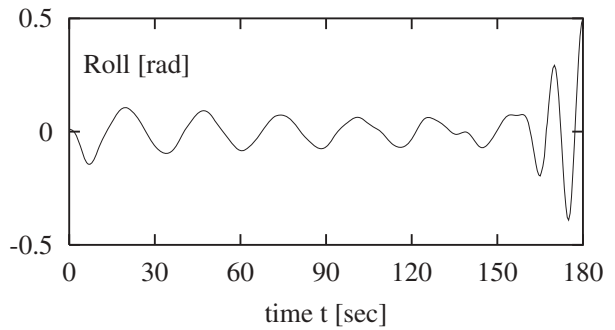

(a)

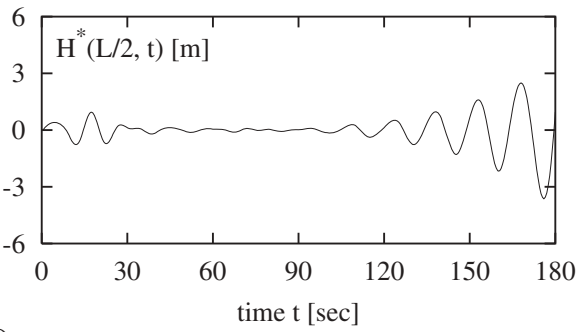

(b)

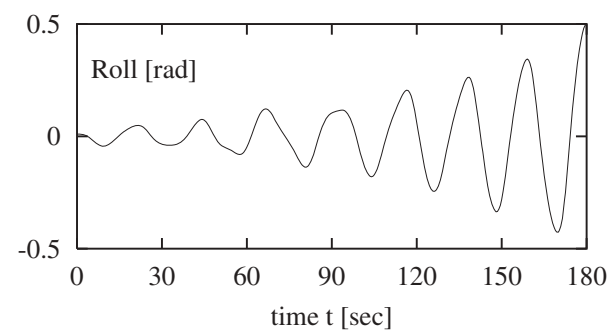

(a)

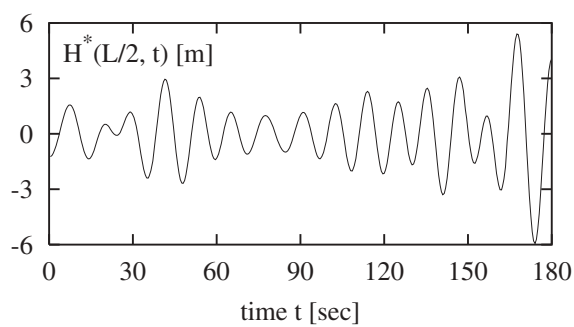

(b)

'regular' wave part in Fig. 6 is slightly higher than that in Fig. 3, whereas the opposite is the case for the transient part. The calculated reliability index $\beta_{F O R M}=3.0447$ is, however, close to the more accurate value 2.9556 associated with the results in Fig. 3. The maximum wave height in the critical wave episode is seen to be around $11 \mathrm{~m}$. Ideally, more frequency components (or nonequidistant steps in the frequency discretization) should have been used, but a few test cases have shown that it does not change $\beta_{F O R M}$ very much even if the critical

waves then look more realistic around $t=0$. distributed frequencies, a head sea, and a forward speed
of the vessel, is slightly less than 180 s. In addition, the $6 \mathrm{~m} / \mathrm{s}, H_{s}=12 \mathrm{~m}, T_{z}=11.7 \mathrm{~s}, t_{0}=180 \mathrm{~s}$, and $n=25$.

The results for the head sea case in Fig. 6 show the same behaviour of parametric roll as in Fig. 3. The main difference is in the beginning of the critical wave episode where an unrealistically high wave is seen in Fig. 6. The reason is that the repetition length of the wave, measured in the coordinate system of the ship, with 25 equally 
For the heading $\psi=150^{\circ}$, Fig. 7 shows also a parametric roll response, but with some initial resonance roll excitation for the first $50 \mathrm{~s}$. The roll resonance period is between $27 \mathrm{~s}$ in still water and $18 \mathrm{~s}$ in large waves. The maximum wave height in the critical wave episode is around $12 \mathrm{~m}$, consistent with a slightly higher reliability index (Fig. 5) than for $\psi=180^{\circ}$.

For the heading $\psi=120^{\circ}$, the results shown in Fig. 8 are significantly different. The most probable roll response shows a very regular pattern for the first $150 \mathrm{~s}$. The period in the response corresponds to the roll period, and the relatively large roll amplitude, given the rather small wave amplitudes in the corresponding critical wave episode, implies that resonance roll is the dominating mode. It is noted that the somewhat irregular critical wave episode actually yields a very regular roll motion. The reason that the regularity in roll is not reflected in the wave generating this response lies mainly in the nonlinearities in the $G Z$ curve in the present model. This somewhat non-realistic behaviour is probably due to the very simplistic motion model employed, especially the omission of sway and yaw. The roll behaviour for the last $30 \mathrm{~s}$ before the maximum roll angle is experienced looks more like forced roll, as the period in the roll response is now much closer to the dominating encounter period of around $11 \mathrm{~s}$. Furthermore, the wave height increases significantly with a maximum wave height of around $18 \mathrm{~m}$. This increase in wave height compared to the case $\psi=150^{\circ}$ is again reflected in a larger reliability index, even if the shorter memory effect ( $30 \mathrm{~s}$ as compared to $180 \mathrm{~s}$ ) tends toward a lower reliability index.

For beam seas, as shown in Fig. 9, roll resonance also dominates for the first $160 \mathrm{~s}$ of the most probable response; the amplitudes are, however, only about half of those seen for $\psi=120^{\circ}$. The last $20 \mathrm{~s}$ is again forced roll with a period close to the dominating encounter period. While the roll response is rather similar to the case $\psi=120^{\circ}$, the corresponding critical wave episode is very different. It starts with a small transient wave that triggers the roll resonance and then features another larger transient wave for the last $50 \mathrm{~s}$. The memory in the system is thus very long, yielding a high reliability index considering that the maximum wave height in the critical wave episode is only around $6 \mathrm{~m}$. Again, the result can be questioned due to the simplicity of the motion model.

Finally, Fig. 10 shows the results for $\psi=60^{\circ}$. Here the most probable roll response is clearly resonance excitation throughout. The corresponding critical wave episode has a rather random shape, again reflecting the non-linearities in the system. The maximum wave height is around $11 \mathrm{~m}$ and the reliability index fairly high.
Table 5. Reliability index $\beta$ using the first-order reliability method (FORM) and a Monte Carlo method ( $10^{6}$ simulations)

\begin{tabular}{rll}
\hline Heading $\psi$ & $\beta_{\text {FORM }}$ & $\beta_{\text {Monte Carlo }} 90 \%$ confidence interval \\
\hline $80^{\circ}$ & 3.4090 & $3.4343-3.4884$ \\
$110^{\circ}$ & 2.5764 & $2.6352-2.6526$ \\
$120^{\circ}$ & 2.7716 & $2.9071-2.9316$ \\
$150^{\circ}$ & 2.1093 & $2.1758-2.1865$
\end{tabular}

$\phi_{0}=0.3$ radians, $V=6 \mathrm{~m} / \mathrm{s}, H_{s}=12 \mathrm{~m}, T_{z}=11.7 \mathrm{~s}, n=25$ and $t_{0}=180 \mathrm{~s}$

From the discussion of the results shown in Figs. 6-10 above, it is clearly difficult to guess the shape of the critical wave episode given the most probable roll response. Whether the real optimum has been determined in all cases was checked by running the FORM optimization using different starting points and different search procedures, but no disagreements were found. A rigorous check of the numerical results would be to compare them with simulations using the Monte Carlo method. Due to the computational time required for Monte Carlo simulations, only a few spot checks were made and only for $\phi_{0}=0.3$ radians, see Table 5 . The error in the reliability index as determined by FORM analysis is seen to be within a few per cent. A second-order reliability analysis (SORM) might improve the accuracy of the reliability index, but then Eq. 7 would probably need to be modified accordingly.

It should of course again be stressed that even if the numerical results from the FORM analysis compare well with Monte Carlo simulations, the accuracy of the predicted response depends on the underlying motion model, i.e., Eqs. 12-17. Especially for beam and quartering seas, the influences of pitch, sway, yaw, and (possibly) surge on the roll response can be significant. These motion components are not accounted for in the present model, and better models should definitely be looked at for real applications; the FORM procedure will, however, remain the same.

Finally, the mean out-crossing rate $v\left(\phi_{0}\right)$, given in Eq. 7, and the probability of exceedance within $1 \mathrm{~h}$ $P\left[\max _{T} \phi>\phi_{0} \mid T=3600 s\right]$, given in Eq. 9, are shown in Figs. 11 and 12, respectively, as a function of the heading. The variation of the mean out-crossing rate reflects closely that of the reliability index as shown in Fig. 5, because Eq. 8 is a fairly good approximation in the present case.

Figure 12 shows that, except for heading angles in the range $70^{\circ}-75^{\circ}$, the "warning" level of $\phi_{0}=0.3$ radians will be exceeded within an hour with a probability larger than $50 \%$, whereas, for the "alarm" level of $\phi_{0}=0.5$ radians, this is the case only for heading angles between $150^{\circ}$ and $180^{\circ}$. This is the kind of useful information that 


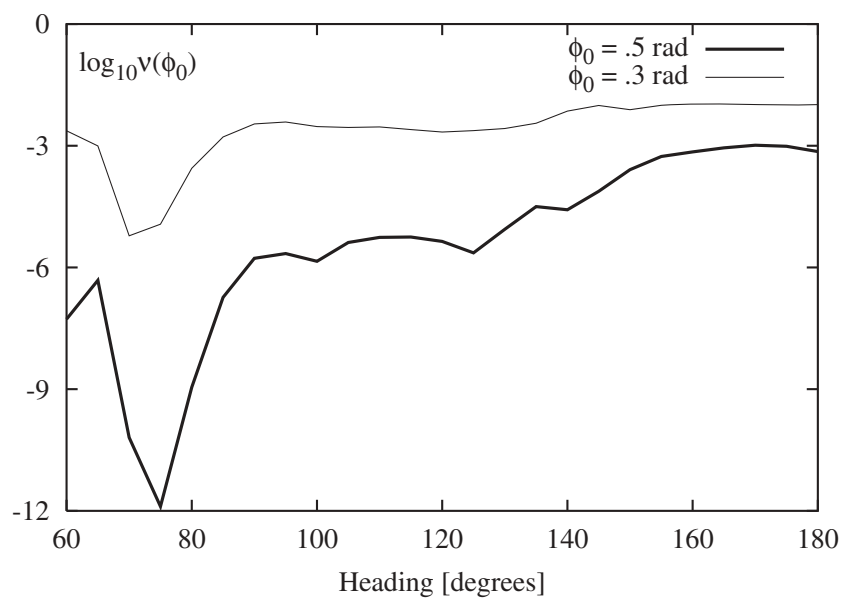

Fig. 11. Mean out-crossing rates as a function of heading angle $\psi$ for $\phi_{0}=0.3$ and 0.5 radians. $V=6 \mathrm{~m} / \mathrm{s}, H_{s}=12 \mathrm{~m}, T_{z}=11.7 \mathrm{~s}, n=$ 25 , and $t_{0}=180 \mathrm{~s}$

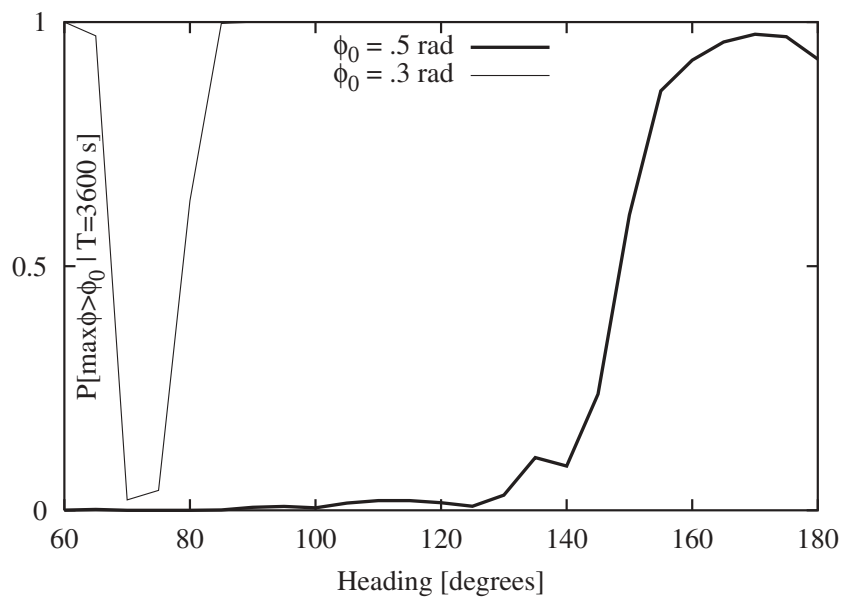

Fig. 12. Probability of exceedance within $1 \mathrm{~h}$ as a function of heading angle $\psi$ for $\phi_{0}=0.3$ and 0.5 radians. $V=6 \mathrm{~m} / \mathrm{s}, H_{s}=12 \mathrm{~m}$, $T_{z}=11.7 \mathrm{~s}, n=25$, and $t_{0}=180 \mathrm{~s}$

the present FORM procedure can deliver to an on-board decision support system. This can be done with reasonable computational effort, but is, of course, conditional on the availability of a proper hydrodynamic code to replace or modify Eq. 12.

\section{Conclusions}

An efficient procedure is presented for the calculation of non-linear extreme responses of marine structures subjected to stationary stochastic wave loads. The first step in the procedure requires a formulation of a time domain description of the response as a function of the wave elevation and wave kinematics. This formulation is then implemented in a standard first-order time-invariant reliability (FORM) code, which for given values of the response can be solved for the associated design points and reliability indices. An analytical expression, Eq. 7, for the mean out-crossing rate in terms of these results is given, and the extreme value distribution of the response is then readily obtained using Eq. 9. Due to the efficient optimisation procedures implemented in standard FORM codes and the short duration needed for the time domain simulations (typically $60-300 \mathrm{~s}$ to cover memory effects in the response), the calculation is very fast. Thus, complicated non-linear effects can be included. The ability of the FORM procedure to deal with very low probabilities of occurrence should also be noted. This is a clear advantage over direct simulation methods.

The procedure was illustrated by application to the roll motion of a ship. Based on a simplified model for the roll motion (Eq. 12), the probability of exceeding a given roll angle within a given time period was calculated for a range of sea states and operational parameters. Such analyses can be done quite fast, thus making the procedure suitable for use in on-board decision support systems to guide the officer on watch, for example.

A number of observations could be made from the parameter study. A general and important one is that the reliability index is exactly inversely proportional to the significant wave height, provided the zero-crossing period is kept the same. Thus a parameter study of the influence of sea state parameters can be restricted to the zero-crossing periods. It was also shown that small changes in speed and/or heading can change the probability of large roll responses significantly in some cases.

It should be stressed again that the present hydrodynamic model, Eq. 12, is a simplified model. It was chosen for the present study because it can represent parametric roll, resonance roll, and forced roll in one model with physically plausible results, at least in near head sea conditions. The inclusion of other ship motions (pitch, sway, yaw, and surge) would require a more elaborate model able to account for the complicated non-linear interactions between the motion components. This is especially important to (auto-generated) parametric roll, dynamic rolling in stern quartering waves, and broaching. A more elaborate hydrodynamic code would of course increase the computational time required for the FORM analysis. The present formulation, Eqs. 12-17, requires around $10 \mathrm{~min}$ of $\mathrm{CPU}$ time per sea state, heading, and speed. Therefore, the model-correctionfactor approach (Ditlevsen and Arnbjerg-Nielsen ${ }^{20}$ ) is probably the most efficient way to improve the results. In this procedure, the critical wave episode determined by the present (or a somewhat modified) simplified for- 
mulation is used as the input to a single deterministic calculation using a more accurate hydrodynamic code. The ratio between the roll angles obtained by the two procedures then acts as a one-to-one mapping between the results.

Acknowledgments. The discussions with Professor Terndrup Pedersen were highly appreciated. The present study was partly supported by the European Commission under the FP6 Sustainable Surface Transport Programme, namely the Integrated Project SAFEDOR (Design, Operation and Regulation for Safety, task 2.3) Contract No. FP6-IP-516278.

\section{References}

1. Rathje H (2005) Impact of extreme waves on ship design and ship operation. In: Proceedings of the conference on design and operation for abnormal conditions. RINA, London

2. Nielsen JK, Hald NH, Michelsen J, et al (2006) SeaSensereal-time onboard decision support. In: Proceedings of the world maritime technology conference, London, March 6-10, IMarEST

3. Borge J, Gonzáles R, Hessner K, et al (2000) Estimation of sea state directional spectra by using marine radar imaging of the sea surface. In: Proceedings of the ETCE/OMAE2000 joint conference, New Orleans, ASME

4. Nielsen UD (2006) Estimations of on-site directional wave spectra from measured ship responses. Mar Struct 19:33-69

5. Krüger S, Hinrichs R, Cramer H (2004) Performance-based approaches for the evaluation of intact stability problems. In: Proceedings of PRADS'2004, Travemünde, September 19(1): 33-69

6. van Daalen EFG, Boonstra H, Blok JJ (2005) Capsize probability analysis of a small container vessel. In: Proceedings of the 8th international workshop on stability and operational safety of ships, Istanbul, October 6-7

7. Bulian G, Francescutto A (2004) A simplified modular approach for the prediction of the roll motion due to the combined action of wind and waves. J Eng Marit Environ 218:189-212

8. Neves MAS, Rodriquez CA (2005) A coupled third-order model of roll parametric resonance. In: Proceedings: maritime transportation and exploitation of ocean and coastal resources. Taylor and Francis, London, pp 243-253

9. Hsieh S-R, Troesch AW, Shaw SW (1994) A nonlinear probabilistic method for predicting vessel capsize in random beam seas. Proc R Soc London, Part A 446:195-211

10. Spyrou KJ (2000) Designing against parametric instability in following seas. Ocean Eng 26:625-653

11. Ness OB, McHenry G, Mathisen J, Winterstein SR (1989) Nonlinear analysis of ship rolling in random beam waves. In: Proceedings of the STAR symposium on 21st century ship and offshore vessel design, production and operation, Society of Naval Architects and Marine Engineers, April 12-15, pp 49-66

12. Søborg AV, Friis-Hansen P (2004) Reliability analysis of dynamic stability in waves. In: Proceedings of the $23 \mathrm{rd}$ international conference on offshore mechanics and arctic engineering (OMAE’04), Vancouver, June, ASME, Paper no. 51591

13. Jensen JJ, Capul J (2006) Extreme response predictions for jack-up units in second-order stochastic waves by FORM. Probab Eng Mech 21(4):330-337

14. Der Kiureghian A (2000) The geometry of random vibrations and solutions by FORM and SORM. Probab Eng Mech 15:81-90

15. Det Norske Veritas (2003) PROBAN, general purpose probabilistic analysis program, version 4.4. Det Norske Veritas, Høvik

16. Lindgren G (1970) Some properties of a normal process near a local maximum. Ann Math Stat 41(6):1870-1883

17. Tromans PS, Anaturk AR, Hagemeijer P (1991) A new model for the kinematics of large ocean waves - application as a design wave. In: Proceedings of the first offshore and polar engineering conference (ISOPE), vol 3, Heriot-Watt University, Edinburgh UK, 11-16 August, International Society of Offshore and Polar Engineers, pp 64-71

18. Adegeest LJM, Braathen A, Løseth RM (1998) Use of nonlinear sea-load simulations in the design of ships. In: Proceedings of PRADS'1998, Delft, Elsevier, pp 53-58

19. Dietz JS, Friis-Hansen P, Jensen JJ (2004) Most likely response waves for estimation of extreme value ship response statistics. In: Proceedings of PRADS'2004, Travemünde, September, Seehafen, Hamburg

20. Ditlevsen O, Arnbjerg-Nielsen T (1994) Model-correctionfactor method in structural reliability. J Eng 120:1-10

21. Alford KA, Troesch AW, McCue LS (2005) Design wave elevations leading to extreme roll motions. In: Proceedings of the 8th international workshop on stability and operational safety of ships, Istanbul, October 6-7

22. Koo H, Der Kiureghian A, Fujimura K (2005) Design point excitation for nonlinear random vibrations. Probab Eng Mech 20(2):136-147

23. Jensen JJ, Pedersen PT (2006) Critical wave episodes for assessment of parametric roll. In: Proceedings of IMDC'06, Ann Arbor, May 16-19, University of Ann Arbor, pp 399-411

24. ITTC Specialist Committee on Stability in Waves (chaired by de Kat JO) (2005) Final report and recommendation. In: Clarke D, Mesbahi AP (eds) Proceedings of the 24th international towing tank conference, University of Newcastle-upon-Tyne, September 4-10, pp 369-408

25. France WN, Levadou M, Treakle TW, et al (2003) An investigation of head-sea parametric rolling and its influence on container lashing systems. Mar Technol 40(1):1-19

26. Shin YS, Belenky VL, Paulling JR, et al (2004) Criteria for parametric roll of large containerships in head seas. Trans SNAME 112:14-47

27. Kroeger H-P (1986) Rollsimulation von schiffen im seegang. Schiffstechnik 33:187-216

28. Jensen JJ, Olsen AS (2006) On the assessment of parametric roll in random seas. In: Proceedings of the world maritime technology conference, London, March 6-10, IMarEST

29. Bulian G (2005) Nonlinear parametric rolling in regular waves - a general procedure for the analytical approximation of the $G Z$ curve and its use in time domain simulations. Ocean Eng 32:309-330

30. Jensen JJ, Mansour AE, Olsen AS (2004) Estimation of ship motions using closed-form expressions. Ocean Eng 31:61-85 